\title{
Error Bounds in Gaussian Integration of Functions of Low-Order Continuity
}

\author{
By Philip Rabinowitz
}

The standard error term in the Gaussian integration rule with $N$ points involves the derivative of order $2 N$ of the integrand. This seems to indicate that such a rule is not efficient for integrating functions of low-order continuity, i.e. functions which have only a few derivatives in the entire interval of integration. However, Stroud and Secrest [3] have shown that Gaussian integration is efficient even in these cases. By applying Peano's theorem [1, p. 109] to functions of loworder continuity, they have tabulated error coefficients $e_{m, N}$ by which the error in integrating such functions can be bounded, provided that a bound $M_{m}$ exists for the derivative of order $m$ of the integrand. In this case,

$$
\left|E_{N}(f)\right|=\left|\int_{-1}^{1} f(x) d x-\sum_{i=1}^{N} w_{i} f\left(x_{i}\right)\right| \leqq e_{m, N} M_{m}
$$

where $\left|f^{(m)}(x)\right| \leqq M_{m}$ in $I=\{-1 \leqq x \leqq 1\}$. In the present paper, we use results from the theory of Chebyshev expansions to compute a different set of error coefficients $d_{m, N}$ which provide sharper bounds on $E_{N}(f)$ in some cases.

Let $f(x)$ be continuous and of bounded variation in $I$. Then there is an expansion of the form

$$
f(x)=\frac{1}{2} a_{0}+a_{1} T_{1}(x)+a_{2} T_{2}(x)+\cdots=\sum_{n=0}^{\infty} a_{n} T_{n}(x)
$$

which is uniformly convergent throughout $I$. Here, $T_{n}(x)$ are the Chebyshev polynomials of the first kind and

$$
a_{n}=\frac{2}{\pi} \int_{-1}^{1} \frac{f(x) T_{n}(x)}{\left(1-x^{2}\right)^{1 / 2}} d x=\frac{2}{\pi} \int_{0}^{\pi} g(\theta) \cos n \theta d \theta
$$

where $g(\theta) \equiv f(\cos \theta)$. By integrating the right-hand integral in (3) successively by parts and applying the second mean-value theorem of the integral calculus after each integration, we get the following results of interest to us. These results as well as additional ones appear in Elliott [2].

A. Define $F_{1}(x) \equiv\left(1-x^{2}\right)^{1 / 2} f^{\prime}(x)$; if $F_{1}(x)$ is of bounded variation in $I$ with $\left|F_{1}(x)\right| \leqq P_{1}$ and if $C_{1}$ is the number of intervals in $I$, in each of which $F_{1}(x)$ is monotonic, then

$$
\left|a_{n}\right| \leqq 4 C_{1} P_{1} / \pi n^{2} \quad \text { for } \quad n \geqq 1 \text {. }
$$

B. Define $F_{2}(x) \equiv\left(1-x^{2}\right) f^{\prime \prime}(x)-x f^{\prime}(x)$; if $F_{2}(x)$ is of bounded variation in $I$ with $\left|F_{2}(x)\right| \leqq P_{2}$, if $C_{2}$ is the number of intervals in $I$, in each of which $F_{2}(x)$ is monotonic, and if $\lim _{x \rightarrow \pm 1} F_{1}(x)=0$, then

Received July 10, 1967. 


$$
\left|a_{n}\right| \leqq 4 C_{2} P_{2} / \pi n^{3} \text { for } n \geqq 1 .
$$

Let us now apply the operator $E_{N}$ to (2). We get

$$
E_{N}(f)=E_{N}\left(\sum_{n=0}^{\infty} a_{n} a_{n}(x)\right)=\sum_{n=0}^{\infty} a_{n}^{\prime} E_{N}\left(T_{n}\right)=\sum_{n=2 N}^{\infty} a_{n} E_{N}\left(T_{n}\right)
$$

since $E_{N}\left(T_{n}\right)=0$ for $n<2 N$. If now $f(x)$ satisfies the conditions A, we get

$$
\left|E_{N}(f)\right| \leqq \frac{4 C_{1} P_{1}}{\pi} \sum_{n=2 N}^{\infty} \frac{\left|E_{N}\left(T_{n}\right)\right|}{n^{2}}=d_{1, N} C_{1} P_{1}
$$

where

$$
d_{1, N}=\frac{4}{\pi} \sum_{n=2 N}^{\infty} \frac{\left|E_{N}\left(T_{n}\right)\right|}{n^{2}}
$$

converges since $\left|E_{N}\left(T_{n}\right)\right| \leqq 2+2 /\left(n^{2}-1\right)$. This bound holds since $\left|T_{n}(x)\right| \leqq 1$ in $I$ and $\sum_{i=1}^{N} w_{i}=2$ implying that $\left|\sum_{i=1}^{N} w_{i} T_{n}\left(x_{i}\right)\right| \leqq 2$ and since $\int_{-1}^{1} T_{n}(x) d x$ $=2 /\left(n^{2}-1\right)$. If $f(x)$ satisfies conditions $\mathrm{B}$, we get similarly

$$
\left|E_{N}(f)\right| \leqq d_{2, N} C_{2} P_{2}
$$

where

$$
d_{2, N}=\frac{4}{\pi} \sum_{n=2 N}^{\infty} \frac{\left|E_{N}\left(T_{n}\right)\right|}{n^{3}} .
$$

In Table 1, values of $e_{i, N}$ and $d_{i, N}$ are given for $i=1,2$ and $N=4(3) 16$. We see that $d_{i, N} / e_{i, N}<1$ and that this ratio decreases with increasing $N$. Hence, in cases where $C_{i} P_{i}$ is not too much greater than $M_{i}$, (7) and (9) will provide sharper error bounds than (1), especially for large $N$.

\section{TABLE 1}

\begin{tabular}{rcccc}
\hline$N$ & $e_{1, N}$ & $d_{1, N}$ & $e_{2, N}$ & $d_{2, N}$ \\
\hline 4 & $2.76(-1)$ & $8.64(-2)$ & $2.19(-2)$ & $7.07(-3)$ \\
7 & $1.65(-1)$ & $3.13(-2)$ & $7.63(-3)$ & $1.50(-3)$ \\
10 & $1.18(-1)$ & $1.60(-2)$ & $3.86(-3)$ & $5.40(-4)$ \\
13 & $9.15(-2)$ & $9.68(-3)$ & $2.33(-3)$ & $2.54(-4)$ \\
16 & $7.48(-2)$ & $6.48(-3)$ & $1.56(-3)$ & $1.39(-4)$ \\
\hline
\end{tabular}

Examples. 1. $f(x)=|x|^{4 / 3}$. In this case, $f^{\prime \prime}(x)$ is unbounded in $I$ so that using (1), we find $E_{N}(f) \leqq e_{1, N} M_{1}$. Taking $N=16$ and $M_{1}=4 / 3$, we find $E_{16}(f)$ $\leqq 1.0(-1)$. Using (7) with $C_{1}=3$ and $P_{1}=.92$, we find $E_{16}(f) \leqq 1.8(-2)$. The actual error is $1.0(-3)$. For $N=4$, the figures are $3.7(-1), 2.4(-1)$, and $2.2(-2)$, respectively.

2. $f(x)=|x|^{8 / 3}$. In this case, $E_{N}(f) \leqq e_{2, N} M_{2}$. With $N=16$ and $M_{2}=40 / 9$, we find $E_{16}(f) \leqq 7.0(-3)$. Using (9) with $C_{2}=3$ and $P_{2}=8 / 3$, we find $E_{16}(f) \leqq 1.2(-3)$. The actual error is $3.5(-5)$. For $N=4$, the figures are $9.8(-2)$, $5.7(-2)$ and $5.1(-3)$, respectively. 
3. $f(x)=(x+1)^{5 / 4}$. In this case also, $f^{\prime \prime}(x)$ is unbounded in $I$ so that $E_{N}(f)$ $\leqq e_{1, N} M_{1}$. With $N=16$ and $M_{1}=(5 / 4) 2^{1 / 4}$ we find $E_{16}(f) \leqq 1.1(-1)$. However, $F_{2}(x)$ satisfies conditions B so that we can use (9). With $C_{2}=2$ and $P_{2}=(5 / 4) 2^{1 / 4}$, we find $E_{16}(f) \leqq 4.2(-4)$. The actual error is $8.9(-7)$.

Remarks. 1. This method is not restricted to Gaussian rules but is applicable to any integration rule defined over $I$ which integrates constants exactly. This includes the Lobatto, Radau, Newton-Cotes, Romberg and Gauss-Jacobi rules.

2. This method can be extended to cases where higher derivatives exist. Thus, Elliott [2] gives the estimate $\left|a_{n}\right| \leqq 4 C_{3} P_{3} / \pi n^{4}$ where

$$
F_{3}(x) \equiv\left(1-x^{2}\right)^{1 / 2}\left[\left(1-x^{2}\right) f^{\prime \prime \prime}(x)-3 x f^{\prime \prime}(x)-f^{\prime}(x)\right]
$$

satisfies conditions similar to $\mathrm{B}$. However, the expressions for $F_{i}$ become very complicated with increasing $i$ and it is not worth the effort to find $C_{i}$ and $P_{i}$.

3. Elliott also gives the estimate $\left|a_{n}\right| \leqq 4 C_{0} P_{0} / \pi n$ where $F_{0}(x) \equiv f(x)$. However, it is probably not possible to use this method for functions with unbounded first derivatives. This is so since $\sum_{n=2 N}^{\infty}\left|E_{N}\left(T_{n}\right)\right| / n$ probably diverges. This assumption is based on the fact that for Gauss-Chebyshev integration, we can prove divergence. The Gauss-Chebyshev integration rule is of the form

$$
\int_{-1}^{1} \frac{f(x)}{\left(1-x^{2}\right)^{1 / 2}} d x=\frac{\pi}{N} \sum_{i=1}^{N} f\left(x_{i}\right)+E_{N}(f)
$$

where

$$
x_{i}=\cos \frac{4 \imath-1}{2 N} \pi, \quad i=1, \cdots, N .
$$

Since $\int_{-1}^{1} T_{n}(x) /\left(1-x^{2}\right)^{1 / 2} d x=0$ for $n \geqq 1$, it follows that $E_{N}\left(T_{n}\right)$ $=(\pi / N) \quad \sum_{i=1}^{N} T_{n}\left(x_{i}\right)$. Since $T_{n}(x)=\cos (n \arccos x)$, we have $T_{n}\left(x_{i}\right)$ $=\cos ((2 i-1) n \pi / 2 N)$. Hence, for $n=2 K N, K=1,2, \cdots, E_{N}\left(T_{n}\right)=-\pi$, from which it follows that $\sum_{n=2 N}^{\infty}\left|E_{N}\left(T_{n}\right)\right| / n$ diverges.

Conclusions. As Examples 1 and 2 indicate, error bounds (1), (7) and (9) may give rather good bounds on the integration error. On the other hand, Example 3 shows that the bounds may overshoot the actual error by many orders of magnitude. Nevertheless, in the absence of further information, they are the best available for functions of low-order continuity. Since $\left|F_{1}(x)\right| \leqq\left|f^{\prime}(x)\right|$ in $I$, (7) will be better than (1) for small values of $C_{1}$. The situation with $F_{2}$ is more complicated but usually $P_{2}$ will be of the same order of magnitude as $M_{2}$ so that (9) will give a better bound than (1) for small values of $C_{2}$. In both cases, the critical value of $C_{i}$ increases with $N$. In cases when the singularity is at an endpoint of $I$, our method may be very advantageous. As Example 3 shows, we can use (9) even when $f^{\prime \prime}(x)$ is unbounded. More generally, $f^{(j)}(x)$ may be unbounded while $F_{j+k}(x)$ is well behaved, $k=0,1, \cdots$. But as mentioned above, the work involved in calculating $C_{j+k}$ and $P_{j+k}$ becomes prohibitive. On the other hand, (1) has the advantage of simplicity especially when compared with (9), and, of course, (1) is preferable when $C_{i}$ is large. Hence there is room for both types of error bound. 
1. P. J. Davis \& P. Rabinowitz, Numerical Integration, Blaisdell, Waltham, Mass., 1967.

2. D. Elliotr, "A Chebyshev series method for the numerical solution of Fredholm integral equations," Comput. J., v. 6, 1963, pp. 102-111. MR 27 \#5386.

3. A. H. Stroud \& D. Secrest, Gaussian Quadrature Formulas, Prentice-Hall, Englewood Cliffs, N. J., 1966. MR 34 \#2185.

\title{
An Explicit Sixth-Order Runge-Kutta Formula
}

\author{
By H. A. Luther
}

1. Introduction. The system of ordinary differential equations considered has the form

$$
d y / d x=f(x, y), \quad y\left(x_{0}\right)=y_{0} .
$$

Here $y(x)$ and $f(x, y)$ are vector-valued functions

$$
\begin{aligned}
y(x) & =\left(y_{1}(x), y_{2}(x), \cdots, y_{m}(x)\right), \\
f(x, y) & =\left(f_{1}(x, y), f_{2}(x, y), \cdots, f_{m}(x, y)\right),
\end{aligned}
$$

so that we are dealing with $m$ simultaneous first-order equations.

For the fifth-order case, explicit Runge-Kutta formulas have been found whose remainder, while of order six when $y$ is present in (1), does become of order seven when $f$ is a function of $x$ alone [3], [4]. This is due to the use of six functional substitutions, a necessary feature when $y$ occurs nontrivially [1].

A family of explicit sixth-order formulas has been described [1]. In this family is the formula given in the next section. Its remainder, while of order seven when $y$ is present in (1), is of order eight when $f$ is a function of $x$ alone. Here again the possibility arises because seven functional substitutions are used, rather than six. Once more, this is a necessity [2].

For selected equations (those not strongly dependent on $y$ ) such formulas seem to lead to some increase in accuracy.

2. Presentation of the Formula. For the interval $\left[x_{n}, x_{n}+h\right]$, Lobatto quadrature points leading to a remainder of order eight are

$x_{n}, \quad x_{n}+h / 2, \quad x_{n}+\left(7-(21)^{1 / 2}\right) h / 14, \quad x_{n}+\left(7+(21)^{1 / 2}\right) h / 14, \quad x_{n}+h$.

A set of Runge-Kutta formulas related thereto is given below. They can be verified by substitution in the relations given by Butcher [1].

Expressed in a usual form they are

Received December 28, 1966. Revised July 31, 1967. 